\title{
Polyomaviridae assembly polymorphism from an energy landscape perspective
}

\author{
Karim M. ElSawy ${ }^{\mathrm{a}, \mathrm{b}}$, Leo S.D. Caves ${ }^{\mathrm{a}, \mathrm{b}}$ and Reidun Twarock ${ }^{\mathrm{a}, \mathrm{b}, \mathrm{c} *}$ \\ ${ }^{a}$ York Centre for Complex Systems Analysis (YCCSA), University of York, York, UK, ${ }^{b}$ Department of \\ Biology, University of York, York, UK, ${ }^{c}$ Department of Mathematics, University of York, York, UK
}

(Received 2 April 2008; final version received 26 April 2008)

\begin{abstract}
Polyomaviridae assemble in vitro into different aggregates depending on experimental conditions. We use an energy landscape approach using empirical energy calculations to quantify how the formation of these different aggregates depends on $\mathrm{pH}$, the presence of bound calcium ions and disulfide linkages. Computations are carried out for SV40, a member of the Polyomaviridae family and are based on the binding free energy landscape of three distinct trimers of pentamers that correspond to the different bonding configurations between the capsid proteins observed in its crystal structure. Our computational analysis shows that the energetics of one of these environments is pivotal for the polymorphic assembly behaviour of SV40, whilst the binding energy landscapes of the other two environments are broadly funnelshaped and thus contribute little to the formation of particles other than virus-like particles (VLP). We have quantified how the existence of bound calcium ions in the absence of disulfide linkages enhances the binding free energies of all three environments and hence, favours the assembly of VLPs. Moreover, estimation of the relative binding free energies of the three environments at $\mathrm{pH} 5$ and $\mathrm{pH} 8$ reveals that they are destabilized at $\mathrm{pH} 5$ relative to $\mathrm{pH}$. The extent of this destabilization is dependent on the presence of disulfide linkages and bound calcium ions and accounts for the experimentally observed polymorphic behaviour of VP1 proteins at $\mathrm{pH} 5$. Interestingly, concurrent existence of bound calcium ions and disulfide linkages is found to be destabilizing and thus may disrupt the assembly of VLPs at pH 8 .
\end{abstract}

Keywords: energy landscape; viral tiling theory; assembly polymorphism; SV40

\section{Introduction}

The assembly of the protein containers called viral capsids, that encapsulate and hence, provide protection for the viral genome has attracted considerable attention, and models have been proposed for the thermodynamic and kinetic [9,15,25] aspects of assembly. A common challenge in the modelling of capsid assembly is the large number of transient intermediate structures [14] and strategies have been devised to reduce the number of assembly pathways that need to be considered $[15,20]$. Since a full atomistic model is difficult to implement given the size of the viral capsids, assembly models have been discussed at various levels of coarsegraining $[3,25,28,39]$. In this work, we present a full atomistic approach to the early stages of the viral assembly process. We show that the energy landscapes associated with aggregates at the early stages of assembly provide valuable information on the spectrum of the final products and hence, permit the study of assembly polymorphism.

We focus on simian virus 40 (SV40), a member of the Polyomaviridae, which include a number of cancer-causing viruses. A distinguishing feature of viruses in this family is the

\footnotetext{
*Corresponding author. Email: rt507@york.ac.uk
} 
unusual organization of their capsid proteins: They occur uniquely in clusters of five called pentamers, as opposed to the commonly observed mix of 12 pentamers and otherwise, hexamers (clusters of six) predicted by Caspar-Klug theory [5]. In particular, the viral capsid of SV40 is composed of 72 pentamers of the VP1 protein that are arranged with icosahedral symmetry [29]. The assembly of the viral capsid of SV40 from its protein building blocks has been studied from both a theoretical $[4,5,20,38]$ and an experimental $[2,10,18,40]$ point of view. It has been shown that the recombinant VP1 protein assembles in insect cells into VLPs in the absence of other virion constituents [23], and that such VLPs are morphologically indistinguishable from the wild-type SV40. SV40 is hence a useful system for studying virus (dis-)assembly [30].

It has been shown that the pentamers self-assemble in vitro into a variety of polymorphic aggregates of spherical or tubular particles depending on experimental conditions such as $\mathrm{pH}$, calcium ion concentration or ionic strength [19,31]. Moreover, reducing agents have been found to disrupt the assembly of SV40 [6] leading to the proposal that disulfide linkages stabilize the viral capsids. Notably, no disulfide linkages exist within SV40 pentamers. However, X-ray diffraction experiments of mercury-labelled SV40 crystals suggested that VP1 cysteines at positions 9, 104 and 207 may be involved in inter-pentamer disulfide linkage formation [24], and a systematic mutagenesis of cysteine to serine codons in the SV40 virion moreover suggests that the formation of post-pentameric complexes is stabilized by the formation of inter-pentamer disulfide linkages [18]. Currently, the formation of disulfide linkages is believed to take place after completion of capsid assembly [34] and we are interested in quantifying via an energy landscape approach, whether this is indeed the favourable scenario.

Therefore, we study here the dependence of the early stages of assembly on $\mathrm{pH}$, calcium ion concentration and the presence of disulfide bridges. In particular, we use an energy landscape approach to investigate the role of disulfide linkages and bound calcium ions in the assembly

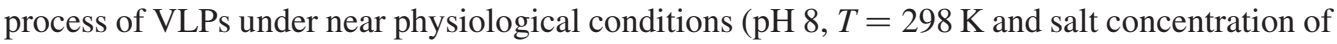
$0.15 \mathrm{M}$ ). Conditions favouring the formation of tubular and medium-size particles are inferred and the stabilizing/destabilizing effect of $\mathrm{pH}$ on the aggregates is discussed.

\section{Estimating the relative free energy landscape of virus pentamer packing in different possible trimer configurations}

The size and shape of the assembled particles depends largely on the packing angles between the pentamers which are fairly rigid and flat. Based on the assumption that the decision between different final products of assembly is made at the early stages of assembly when, for example for spherical particles, the radius and curvature of the particles are fixed, we choose to focus our analysis on a trimer of pentamers. These are aggregates formed from 15 VP1 capsid proteins, later referred to as trimers for brevity. Viral Tiling Theory (VTT) shows that there are three different bonding environments (configurations) between such trimers in the capsid of SV40 $[20,22]$ as shown in Figure 1(a)-(c). Experimental data on the structures of each of these three trimers were extracted from the coordinate (PDB) file of the X-ray crystal structure of SV40 [34]. The conformational space of each of the three trimers was represented via the packing angles $\gamma 1$ and $\gamma 2$, that represent the relative orientations of these pentamers as shown in Figure $1(d)$ and serve as collective degrees of freedom.

The variation of the binding free energy between pentamers in the trimer units within this space gives rise to the binding free landscape, which can be utilized for studying the relative stabilities of the assembled particles. The potential energy surface (PES) of each of the three trimers is computed relative to a grid in the $(\gamma 1, \gamma 2)$ space with grid resolution of $5^{\circ}$ over the range $0-60^{\circ}$ for both angles. These search limits were chosen so as to encompass the range of observed structures at experimental conditions. The coordinates of the atomic positions of the 
(a)
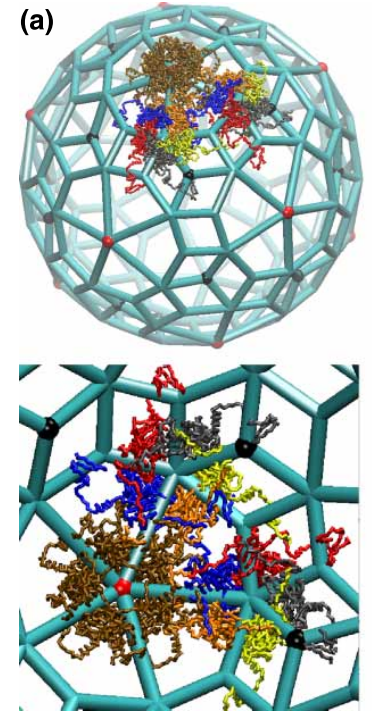

(b)
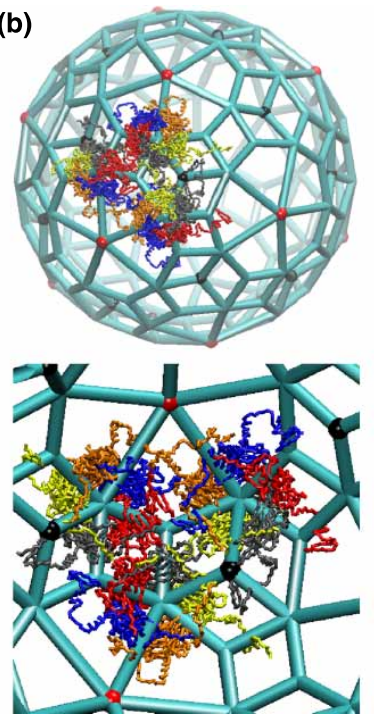

(c)
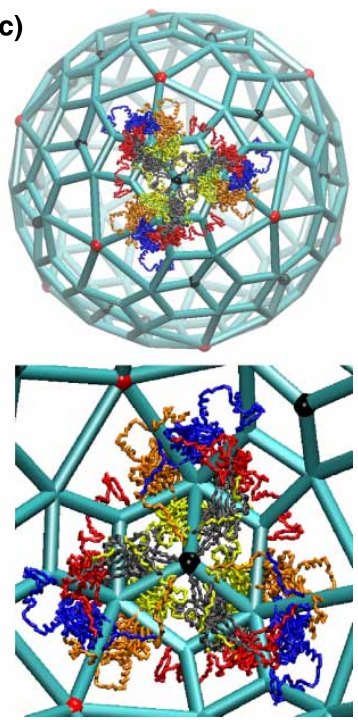

(d)

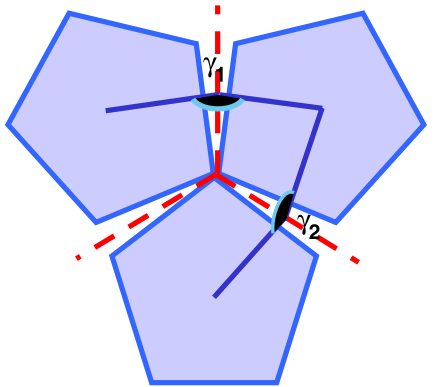

Figure 1. The three distinct molecular aggregates within SV40 X-ray structure superposed on the tiling scheme proposed by the virus tiling theory $(\mathrm{a}-\mathrm{c})$ and the definition of $\gamma 1$ and $\gamma 2$ packing angles (d). (a) Trimer interaction (trimer 1), (b) trimer-dimer interaction (trimer 2) while, (c) dimer interaction (trimer 3). The lower panel corresponds to a close-up view of the trimers from inside SV40 capsid. The 5-fold symmetry axis is shown as a red sphere while the 3 -fold axis is shown in black. Available in colour online.

proteins at each grid point were obtained by rotation around the lines of intersection of the partial least square regression planes passing through neighbouring pentamers. The PESs for each trimer were computed at two $\mathrm{pH}$ values (5 and 8 ) by adjusting the protonation states of ionizable residues using the PROPKA algorithm [16]. For each $\mathrm{pH}$ value, the potential energy of the structures was calculated using the CHARMM22 force field [3]. A relative dielectric constant of 12 was used for the electrostatic term, consistent with experimental $\mathrm{pK}_{\mathrm{a}}$ measurement of buried residues [11] and subsequent continuum model calculation of solvation energies [32] in analogous protein systems. In order to relieve the steric contacts, introduced at the pentamerpentamer interface by the search procedure, the system was subjected to three consecutive rounds of steepest descent energy minimization whilst the backbone atoms were under harmonic restraint using an initial force constant of $30 \mathrm{kcal} \mathrm{mol}^{-1} \AA^{-2}$. The force constant was decreased by $10 \mathrm{kcal} \mathrm{mol}^{-1} \AA^{-2}$ after each round. After this minimization step the root mean square difference (RMSD) of the backbone atoms was about $0.3 \AA$ relative to the initial structure. In order to capture the effect of disulfide linkage formation and bound calcium ions, the above 
minimization protocol was repeated for possible combinations of 'with/without calcium ions' and 'with/without disulfide linkages'. The presence of a disulfide linkage was determined on the basis of a distance threshold of $2.05 \pm 0.03 \AA$ for the $S-S$ distance of inter-pentamer cystein residues [36]. According to this criterion, no disulfide linkage can form in trimer 1 (Figure 1), whilst they are possible for the other two trimers. The positions of the calcium ions were determined according to [34] based on a maximum distance of $4 \AA$ from Glu216 in one pentamer to Glu330 for the first binding site and to Asp345 for the second binding site in neighbouring pentamers. Distance restraints were applied to these distances during the minimization stage described above.

The binding free energy $\Delta G_{\text {binding }}$ was calculated for each of the three trimers at every point in the $(\gamma 1$ and $\gamma 2)$ conformational space based on the following thermodynamic expression:

$$
\begin{gathered}
\Delta G_{\text {binding }}=\Delta G_{\text {trimer }}-\left(\Delta G_{\text {pentamer } 1}+\Delta G_{\text {pentamer } 2}+\Delta G_{\text {pentamer } 3}\right)+\Delta E_{\mathrm{vdw}} \\
\Delta G=\Delta G_{\text {total.solv }}=\Delta G_{\text {elec }}+\Delta G_{\text {apolar }} .
\end{gathered}
$$

The total solvation free energy $\Delta G_{\text {total.solv }}$ can be expressed as a sum of electrostatic $\Delta G_{\text {elec }}$ and non polar $\Delta G_{\text {apolar }}$ components [33]. The electrostatic component was calculated based on a finite difference solution of the linearized Poisson-Boltzmann equation [27] using the APBS package [1]. A final grid spacing of $0.5 \AA$ was used throughout the study. The solvent and protein relative dielectric constants were set to 78.54 and 12 respectively, while the salt concentration was set to $0.15 \mathrm{M}$. The non-polar component was calculated as a solventaccessible surface area (SASA) dependent term where each $\AA^{2}$ of SASA contributes $5 \mathrm{cal} \mathrm{mol}^{-1} \AA^{-2}$ to the solvation free energy [35]. The change in the van der Waals component of the potential energy $\Delta E_{\mathrm{vdw}}$ was extracted from the potential energy landscape computed using the CHARMM22 force field [3].

We note that this study of the viral assembly process in terms of the binding free energy landscape differs from other approaches such as the molecular dynamics simulations in Refs. $[14,25]$, which are inherently dependent on the conformational sampling generated in the time domain. In the method adopted in this study, the search procedure explicitly defines the limits of the conformational space to be explored and circumvents timescale issues. Such an approach has proved useful in studying DNA [8] and protein [12] energy landscapes.

Estimation of the absolute binding free energies of protein-protein association $(\Delta G)$ is recognized to be very difficult, requiring careful attention to issues such as parameterization of SASA potential, estimation of conformational entropy [13]. In this initial study, we do not place emphasis on the estimates of the absolute free energies $(\Delta G)$, but use the relative binding free energies $(\Delta \Delta G)$ as a guide to the relative stability of the configurations and conformations of the systems under consideration. Specifically, we examine the general features of the topology of the free energy landscapes, defined in terms of the connectivity of the energy valleys. The energy landscape approach provides a powerful framework for understanding the formation, transformation or decomposition of transient intermediates during the viral assembly process.

\section{Implications for SV40 assembly}

The topologies of the binding free energy landscapes of the three trimers differ significantly in terms of the depth and breadth of their energy valleys (see Figures 2-4). The energy landscapes of trimers 1 and 3 show a broad energy valley in contrast to trimer 2. Such broad energy valleys indicate that the formation of trimers 1 and 3 from their constituent pentamers is quite flexible in terms of the packing angles $\gamma 1$ and $\gamma 2$. Thus, different possible packing arrangements of pentamers are channelled to virtually the same end state, which is very close to the experimentally observed 
(a)

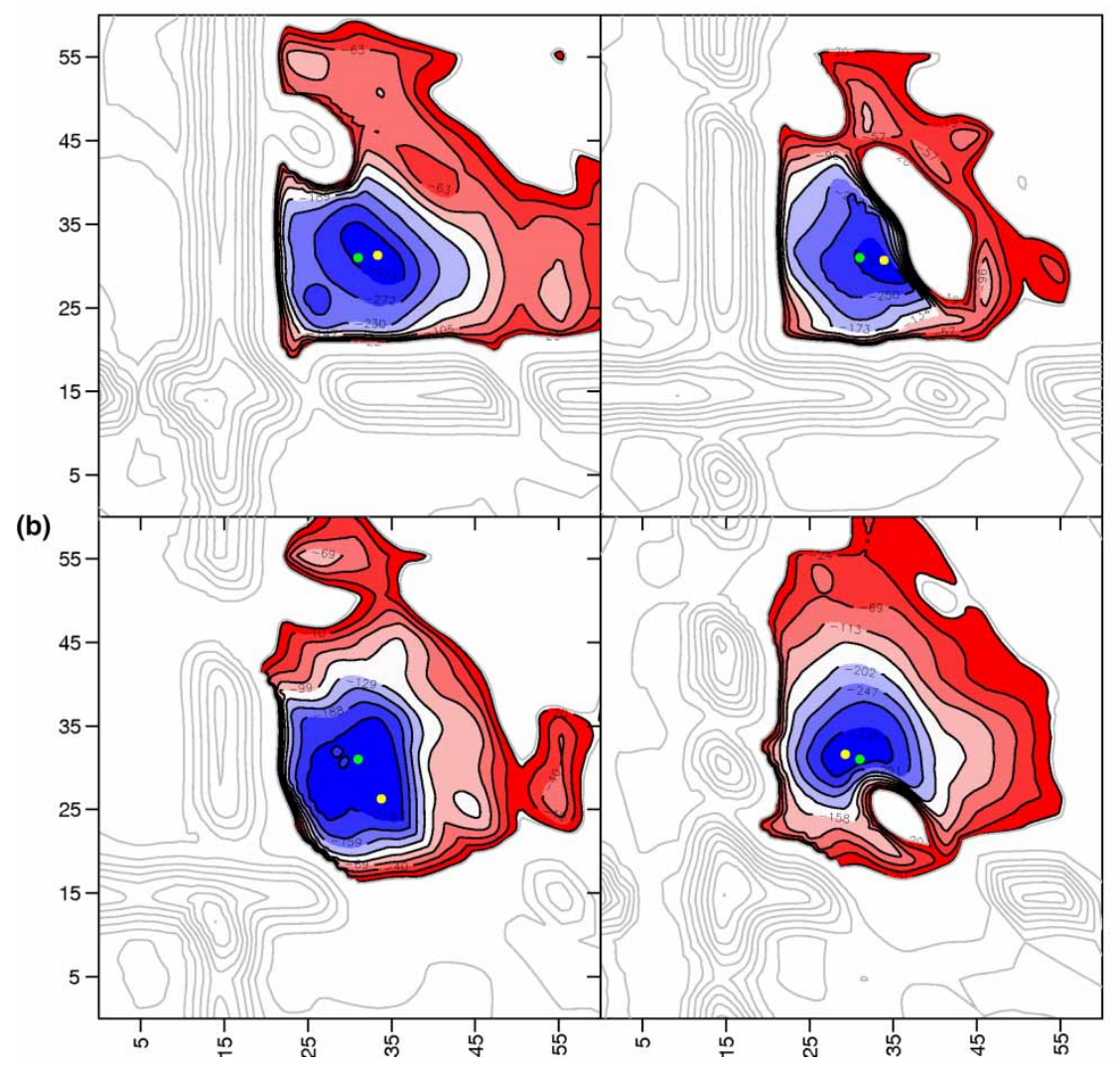

Figure 2. The binding free energy landscapes of trimer 1 at (a) $\mathrm{pH} 5$ and (b) $\mathrm{pH} 8$ within the conformational space defined by the $\gamma 1$ and $\gamma 2$ packing angles. Each panel corresponds to two different cases, row-wise; (without calcium ions) and (with calcium ions). Lowest energy minima are labelled in yellow. The location of SV40 X-ray structure is indicated by a green dot. The colour ramp for the contour lines ranges from deep blue (most stable) to dark red (least stable $\sim-5 \mathrm{kcal} \mathrm{mol}^{-1}$ ). Contours corresponding to positive binding free energy change (unstable configurations) are shown as grey lines. Available in colour online.

structure of SV40 irrespective of $\mathrm{pH}$ or binding of bound calcium ions (Figure 2 and 4 ). The breadth of these energy valleys hence enhances the contribution of the conformational entropy to the binding free energy in each case, which shows that these two trimers do not act as limiting factors on the assembly of the VLPs.

By contrast, the binding free energy landscapes of trimer 2 do not show such a simple topology (Figure 3). The topology of the energy landscape in this case is very much dependent on the $\mathrm{pH}$, existence of calcium ions and disulphide linkages. This is reflected in the varying positions and numbers of the energy minima in each case, see also Table 1 . The narrowness of the energy valleys implies that the binding conformation of trimer 2 is more restrictive in terms of the packing angles $\gamma 1$ and $\gamma 2$. This suggests that trimer 2 is a major factor in SV40 assembly and controls its polymorphic assembly behaviour. This is consistent with the predictions of VTT, which states that the bonding environment represented by the second trimer occurs in the surface structures of tubular or sheet structures (see also Figure 1(b) lower panel; [21,37]), whilst the environments of trimers 1 and 3 contribute only to the formation of VLPs. 
(a)

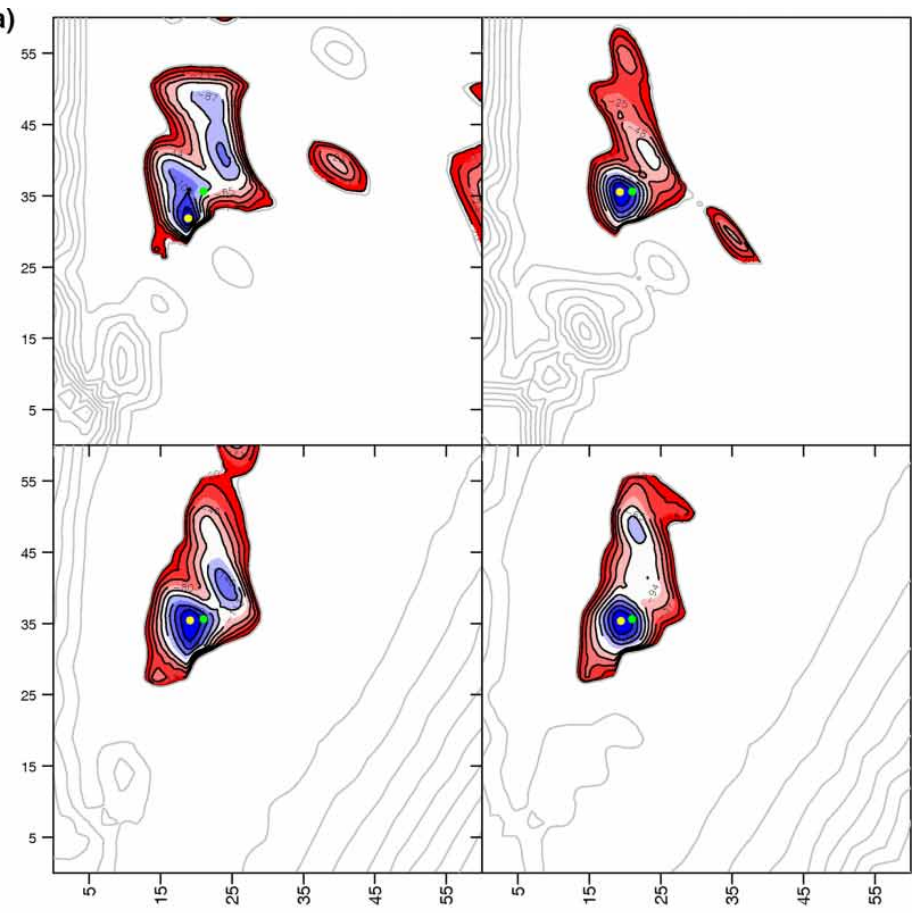

(b)

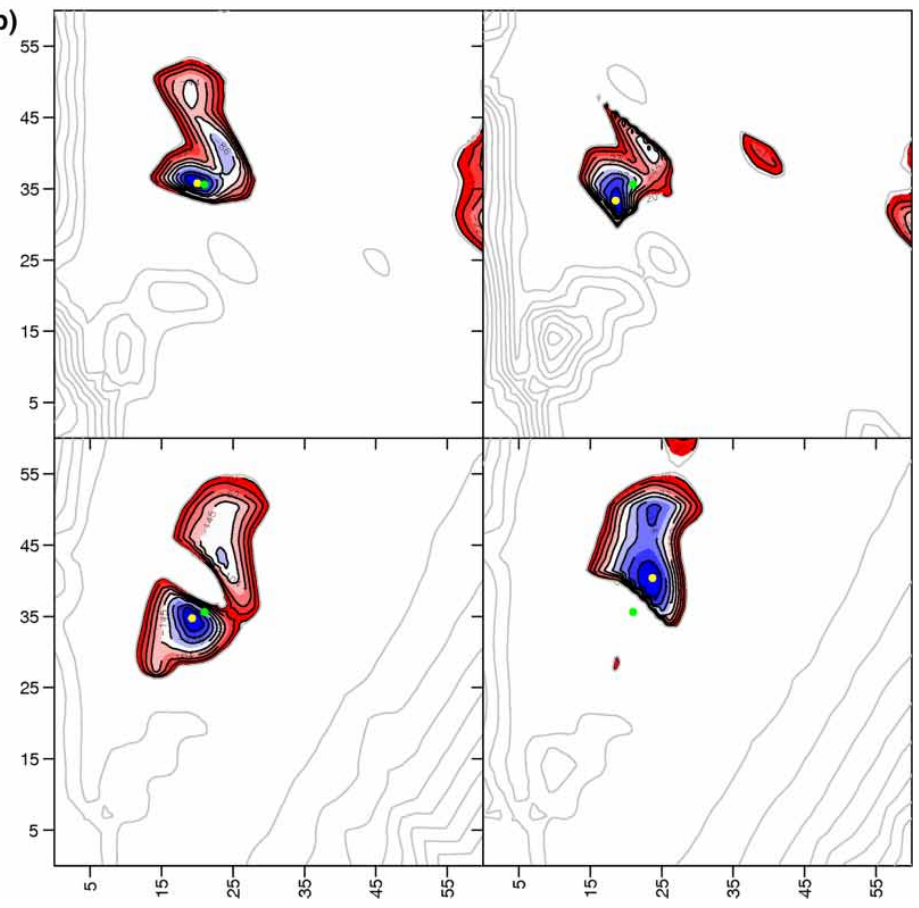

Figure 3. The binding free energy landscapes of trimer 2 at (a) $\mathrm{pH} 5$ and (b) $\mathrm{pH} 8$. Each panel corresponds to four different cases, row-wise: without calcium ions, without disulphide linkages; without calcium ions, with disulphide linkages; with calcium ions, without disulphide linkages; and with calcium ions with disulphide linkages. Lowest energy minima are labelled in yellow while SV40 X-ray structure is in green. Available in colour online. 

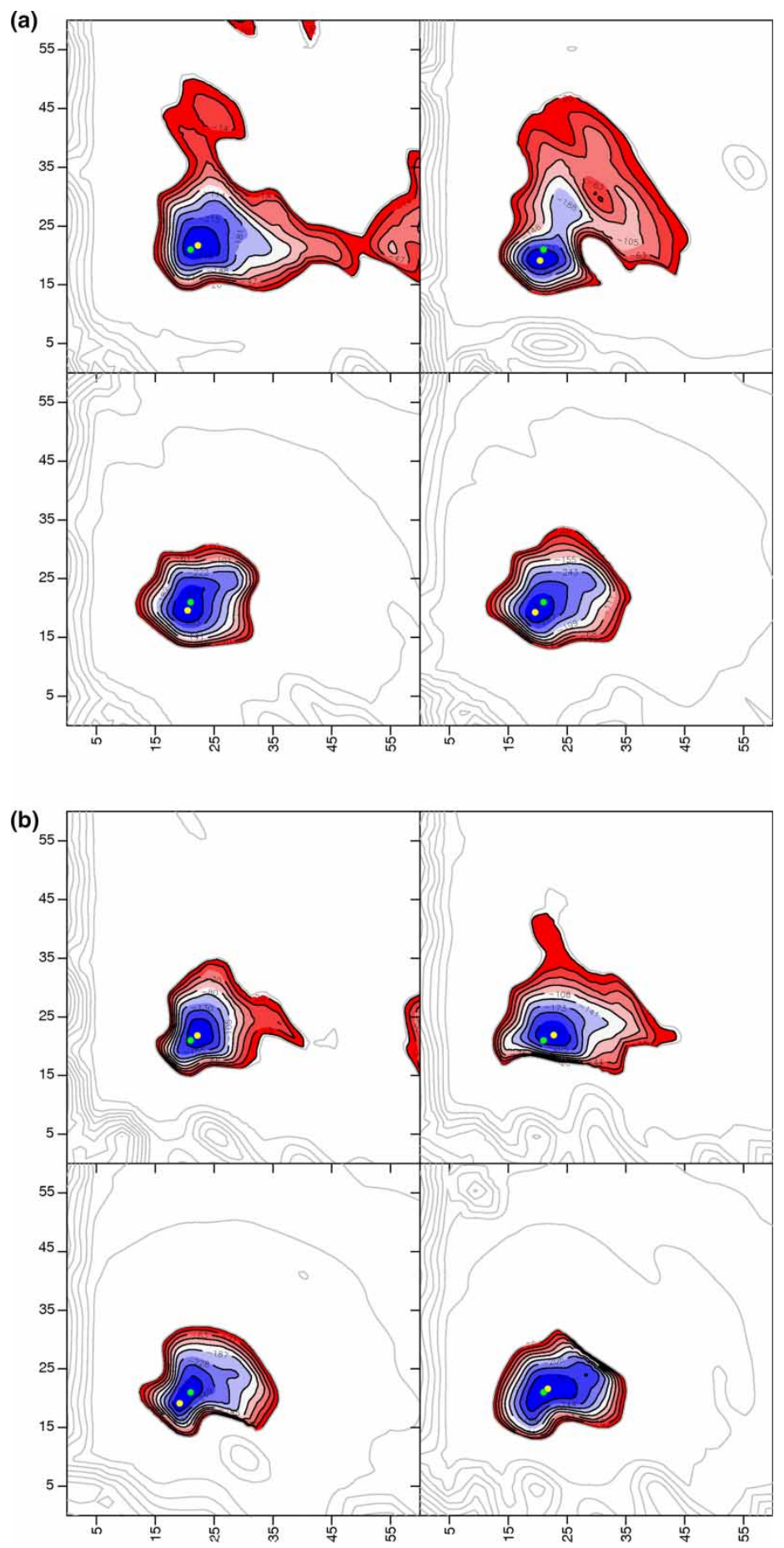

Figure 4. The binding free energy landscapes of trimer 3 at (a) $\mathrm{pH} 5$ and (b) $\mathrm{pH} 8$. Each panel corresponds to four different cases, row-wise: without calcium ions, without disulphide linkages; without calcium ions, with disulphide linkages; with calcium ions, without disulphide linkages; and with calcium ions with disulphide linkages. Available in colour online. 
Table 1. Characteristics of the lowest energy minima on the energy landscape of trimer 2 at $\mathrm{pH} 5$ and $\mathrm{pH} 8$ in the presence and/or absence of calcium ions and disulphide linkages.

\begin{tabular}{|c|c|c|c|c|c|c|c|}
\hline \multirow[b]{2}{*}{ Calcium ions } & \multirow[b]{2}{*}{$\begin{array}{l}\text { Disulfide } \\
\text { linkage }\end{array}$} & \multicolumn{3}{|c|}{$\mathrm{pH} 5$} & \multicolumn{3}{|c|}{$\mathrm{pH} 8$} \\
\hline & & $\begin{array}{c}\Delta G \\
\left(\mathrm{kcal} \mathrm{mol}^{-1}\right)\end{array}$ & $\begin{array}{l}\gamma 1 \\
\left({ }^{\circ}\right)\end{array}$ & $\begin{array}{l}\gamma 2 \\
\left({ }^{\circ}\right)\end{array}$ & $\begin{array}{c}\Delta G \\
\left(\mathrm{kcal} \mathrm{mol}^{-1}\right)\end{array}$ & $\begin{array}{l}\gamma 1 \\
\left({ }^{\circ}\right)\end{array}$ & $\begin{array}{l}\gamma 2 \\
\left({ }^{\circ}\right)\end{array}$ \\
\hline No & No & $\begin{array}{r}-173.3 \\
-111.8 \\
-97.3 \\
-37.3 \\
3.3\end{array}$ & $\begin{array}{l}18.6 \\
16.8 \\
23.2 \\
39.8 \\
14.5\end{array}$ & $\begin{array}{l}31.9 \\
38.5 \\
47.2 \\
39.1 \\
27.5\end{array}$ & $\begin{array}{r}-158.6 \\
-112.6 \\
-72.1\end{array}$ & $\begin{array}{l}19.5 \\
23.2 \\
18.9\end{array}$ & $\begin{array}{l}36.3 \\
41.5 \\
48.4\end{array}$ \\
\hline No & Yes & $\begin{array}{r}-131.6 \\
-74.1 \\
-43.6 \\
-14.1 \\
-5.6 \\
-4.6\end{array}$ & $\begin{array}{l}19.5 \\
23.4 \\
35.1 \\
19.7 \\
17.0 \\
21.0\end{array}$ & $\begin{array}{l}35.1 \\
41.1 \\
29.3 \\
55.4 \\
48.4 \\
53.1\end{array}$ & $\begin{array}{r}-176.3 \\
-104.3 \\
-8.8\end{array}$ & $\begin{array}{l}18.6 \\
23.2 \\
18.6\end{array}$ & $\begin{array}{l}32.8 \\
41.4 \\
44.4\end{array}$ \\
\hline Yes & No & $\begin{array}{r}-274.3 \\
-211.3 \\
-135.3 \\
-58.8\end{array}$ & $\begin{array}{l}19.1 \\
23.5 \\
22.0 \\
14.5\end{array}$ & $\begin{array}{l}34.9 \\
41.2 \\
47.3 \\
27.5\end{array}$ & $\begin{array}{r}-335.3 \\
-195.3 \\
-114.8 \\
-61.8\end{array}$ & $\begin{array}{l}19.0 \\
23.2 \\
25.8 \\
26.1\end{array}$ & $\begin{array}{l}34.8 \\
43.6 \\
39.1 \\
35.7\end{array}$ \\
\hline Yes & Yes & $\begin{array}{r}-227.8 \\
-139.3 \\
-121.8 \\
-14.8\end{array}$ & $\begin{array}{l}19.4 \\
21.8 \\
23.2 \\
28.0\end{array}$ & $\begin{array}{l}35.2 \\
47.5 \\
41.4 \\
50.7\end{array}$ & $\begin{array}{r}-269.1 \\
-217.1 \\
-52.6\end{array}$ & $\begin{array}{l}23.5 \\
23.9 \\
18.6\end{array}$ & $\begin{array}{l}40.7 \\
49.6 \\
28.1\end{array}$ \\
\hline
\end{tabular}

In the following, we study the dependence of the topologies of the energy landscapes for all three trimers on $\mathrm{pH}$, calcium ions and disulfide linkages, and discuss the implications of these results on the stability of VLPs and other polymorphic aggregates.

\subsection{The role of disulfide linkages in stabilizing VLPs}

The packing angles $(\gamma 1$ and $\gamma 2)$ corresponding to the local minima on the binding free energy landscapes in Figures 2-4 are directly related to the curvatures of the trimers that are associated with these minima. Based on the crystal structure of SV40 [34], we determined the $\gamma$ angles corresponding to the three trimer environments as follows: $(\gamma 1, \gamma 2) \sim\left(31^{\circ}, 31^{\circ}\right)$ in trimer $1,(\gamma 1$, $\gamma 2) \sim\left(21^{\circ}, 35^{\circ}\right)$ in trimer 2 and $(\gamma 1, \gamma 2) \sim\left(21^{\circ}, 21^{\circ}\right)$ in trimer 3 . The positions of these pairs on our energy landscape are shown as green dots in Figures 2-4; they are located very close to the bottom of the energy valley. An exception occurs at $\mathrm{pH} 8$ for the case where disulphide linkages are formed while calcium ions are bound (see trimer 2, Figure 3 bottom right corner). This suggests that the $\left(21^{\circ}, 35^{\circ}\right)$ conformation is thermodynamically unstable under these conditions. Interestingly, this conformation is stable in the absence of calcium ions while disulfide linkages are present (Figure 3 bottom left corner). This implies that at $\mathrm{pH} 8$ the existence of bound calcium ions and disulfide linkages is counter-productive. Since all three trimers are required for the formation of the VLPs, the formation of disulphide linkages in the early stages of the assembly process, in the presence of calcium ions, would be disruptive since it destabilizes trimer 2 at the required packing angles. This is consistent with the observation that disulfide linkages are not essential for the formation of VLPs in vivo, however, their formation is critical for maintenance of VLPs at low calcium ion concentrations [17]. 


\subsection{The role of calcium ions at $\mathrm{pH} 8$}

In order to assess quantitatively the major factors affecting the assembly process at $\mathrm{pH} 8$, we focus on the binding free energies $(\Delta G)$ of the most stable trimers in each of the computed energy landscapes, i.e. the conformations corresponding to the lowest binding free energy minima in Figures 2-4 bottom panels (Table 2). The binding free energies of these trimers reveal the importance of calcium ions for the assembly process. For example, the binding free energy of trimer 2 in the presence of calcium ions and absence of disulfide linkages is about $-335.8 \mathrm{kcal} \mathrm{mol}^{-1}$, which is almost twice that of its counterpart in the absence of calcium ions (about $-158 \mathrm{kcal} \mathrm{mol}^{-1}$ ). Consistent with our analysis in the previous subsections, this implies that the most stable trimers correspond to states where bound calcium ions are present in the absence of disulfide linkages and the packing angles distinguished by the energy surface are in good agreement with those in the crystal structure of SV40. By contrast, the most stable conformations in the presence of bound calcium ions and disulphide linkages deviates from the angles in the observed SV40 structure for the case of trimer 2, further corroborating that the disulphide linkages are not formed during the early stages of the assembly process of SV40 [19,31].

\subsection{Binding affinities at $\mathrm{pH} 5$ relative to $\mathrm{pH} 8$}

A change of $\mathrm{pH}$ has significant consequences on the assembly process [19,31]. Variations in the relative stabilities of the three trimers upon changing the $\mathrm{pH}$ from $\mathrm{pH} 8$ to $\mathrm{pH} 5$ are indicated by the $\Delta \Delta G$ values in Table 2 . These values indicate that at $\mathrm{pH} 5$, in the absence of calcium ions and disulfide linkages, the three trimers are not equally stabilized relative to their counterparts at $\mathrm{pH}$ 8. Trimer 1 is affected the most by this change in $\mathrm{pH}\left(\Delta \Delta G=-105.5 \mathrm{kcal} \mathrm{mol}^{-1}\right)$, whereas trimer 2 is only slightly affected $\left(\Delta \Delta G=-14.7 \mathrm{kcal} \mathrm{mol}^{-1}\right)$. Changes in the $\mathrm{pH}$ have more dramatic effects under other conditions: For example, in the presence of calcium ions and the absence of disulphide linkages, all three trimers are destabilized. Such a destabilization of one or all of the three trimers is expected to alter the assembly pathway from the production of VLPs to the formation of other aggregates. This is consistent with the observation of tubular particles at pH 5 rather than VLPs [19]. An analysis of the specific morphologies of these tubular aggregates via our energy landscape approach will be published elsewhere [7].

Table 2. Relative free energies of binding $(\Delta \Delta G)$ at $\mathrm{pH} 5$ vs. $\mathrm{pH} 8$ for the most stable conformations $(\gamma 1$ and $\gamma 2)$ of the three trimer configurations. The significance of the reported energies is indicative and for purposes of comparison. A statistical estimate of the precision requires a more extensive sampling of the conformational space which is the subject of ongoing work.

\begin{tabular}{|c|c|c|c|c|c|c|c|c|c|}
\hline \multirow{2}{*}{$\frac{\text { Trimer }}{1}$} & \multirow{2}{*}{$\begin{array}{l}\text { Calcium } \\
\text { ions }\end{array}$} & \multirow{2}{*}{$\begin{array}{l}\text { Disulfides } \\
\text { No }\end{array}$} & \multirow{2}{*}{$\begin{array}{c}\begin{array}{l}\Delta G_{\mathrm{pH} 8} 8 \\
(\mathrm{kcal} \\
\left.\mathrm{mol}^{-1}\right)\end{array} \\
-245.6\end{array}$} & \multirow{2}{*}{$\begin{array}{l}\Delta G_{\mathrm{pH} 5} \\
(\mathrm{kcal} \\
\left.\mathrm{mol}^{-1}\right)\end{array}$} & \multirow{2}{*}{$\begin{array}{l}* \Delta \Delta G \\
\begin{array}{l}(\mathrm{kcal} \\
\left.\mathrm{mol}^{-1}\right)\end{array} \\
-105.5\end{array}$} & \multicolumn{2}{|c|}{$\begin{array}{c}(\gamma 1, \gamma 2)_{\mathrm{pH}} 8 \\
\left({ }^{\circ}\right)\end{array}$} & \multicolumn{2}{|c|}{$\begin{array}{c}\left(\gamma 1, \underset{\left({ }^{\circ}\right)}{\gamma 2}\right)_{\mathrm{pH} 5} \\
\end{array}$} \\
\hline & & & & & & 33.7 & 26.8 & 33.6 & 31.4 \\
\hline 2 & & & -158.6 & -173.3 & -14.7 & 19.5 & 36.3 & 18.6 & 31.9 \\
\hline 3 & & & -204.7 & -281.3 & -76.6 & 21.5 & 21.3 & 22.4 & 21.9 \\
\hline 2 & No & Yes & -176.3 & -131.6 & 44.6 & 18.6 & 32.8 & 19.5 & 35.1 \\
\hline 3 & & & -267.7 & -352.6 & -84.9 & 22.9 & 21.9 & 20.5 & 19.1 \\
\hline 1 & Yes & No & -383.5 & -322.2 & 61.3 & 30.0 & 31.1 & 33.6 & 30.8 \\
\hline 2 & & & -335.3 & -274.3 & 60.9 & 19.0 & 34.8 & 19.1 & 34.9 \\
\hline 3 & & & -364.4 & -343.0 & 21.4 & 19.8 & 20.0 & 20.4 & 19.8 \\
\hline 2 & Yes & Yes & -269.1 & -227.8 & 41.3 & 23.4 & 40.7 & 19.4 & 35.2 \\
\hline 3 & & & -323.3 & -376.8 & -53.5 & 21.1 & 21.3 & 19.9 & 19.7 \\
\hline
\end{tabular}




\subsection{Assembly of non VLPs}

Different particle shapes have been observed for SV40 depending on experimental conditions. Besides the VLPs which are $400-600 \AA$ in diameter $\left(\gamma\right.$ angles of $\left.\sim 25^{\circ}\right)$, medium-size particles with a diameter of $320 \AA$ ( $\gamma$ angles of $\left.43.7^{\circ}\right)$, small-size particles with a diameter of $260 \AA$ $\left(\gamma\right.$ angles of $\left.63.4^{\circ}\right)$, as well as tubular structures and sheets have been reported [19,31]. According to VTT, trimer 2 is instrumental in the formation of these non-VLP aggregates. For example, VTT suggests that the bonding environment of trimer 2 is the only one required for the formation of the medium-size particles. We therefore investigate the topology of its energy landscape in more detail. Our computations show that the most stable conformation for trimer 2 occurs in the presence of calcium ions and disulfide linkages at $\mathrm{pH} 8$ and corresponds to the packing angles $\left(23.5^{\circ}\right.$ and $\left.40.7^{\circ}\right)$, see Table 1 . Such a $\gamma 2$ value is very close to that of the medium-size particle $\left(\gamma\right.$ angle $\left.43.7^{\circ}\right)$. The $\left(23.5^{\circ}\right.$ and $\left.40.7^{\circ}\right)$ conformation could therefore be an intermediate structure in the assembly of the medium size particle, or an irregular aggregate of a similar size. This is in agreement with the observation that assembly at $\mathrm{pH} 8.5$, in the absence of chelating and reducing agents, results in medium-sized particles and irregular aggregates [34]. Trimer 2 was also predicted by VTT to form small-sized particles [22], which have been experimentally observed at high salt concentration $\left(1 \mathrm{M} \mathrm{NH} \mathrm{NH}_{4} \mathrm{Cl}\right.$; [34]). However, the corresponding conditions are outside the scope of our calculations (conducted at salt concentration of $0.15 \mathrm{M} \mathrm{NaCl}$ ).

\section{Concluding remarks}

In this study, we have analysed assembly polymorphism via an energy landscape approach based on the early stages of the assembly process. A calculation of the binding free energies of the three different bonding environments in the X-ray structure of SV40, in a conformational space defined by the two inter-pentamer packing angles, revealed that the observed polymorphic behaviour of VP1 proteins can be explained via the different topologies of the binding free energy landscapes of the three trimer environments. The formation of trimer 2 has been shown to be pivotal for the polymorphic assembly of SV40, whilst the formation of the other two trimers is not limiting for assembly.

An analysis of the topology of the energy landscape, in the presence or absence of bound calcium ions and/or disulfide linkages at near physiological conditions, revealed interesting results. The concurrent existence of calcium ions and disulfide linkages was found to be counterproductive and could therefore lead to disruption of the progress of the assembly of VLPs. On the other hand, in agreement with experimental results [17], we found that the existence of calcium ions alone enhances the binding free energies of all three environments, with that of trimer 2 almost doubled.

Changing the $\mathrm{pH}$ of the assembly medium proved to have a detrimental effect on the binding free energies of all three environments: At $\mathrm{pH} \mathrm{5,} \mathrm{the} \mathrm{most} \mathrm{stable} \mathrm{conformations} \mathrm{of} \mathrm{the} \mathrm{three}$ environments suffered significant destabilization compared to $\mathrm{pH}$ 8. The magnitude of this destabilization was found to be different for the three environments and affects in particular the trimers necessary for the formation of the VLPs, which are not important for the formation of the other aggregates. This explains in thermodynamic terms the experimentally observed formation of aggregates other than VLP at $\mathrm{pH} 5$.

Our results are in good agreement with the experimentally observed scenarios and provide a framework for quantifying how favourable the different intermediates are from an energy landscape perspective. Moreover, they provide new insights into the role of disulfide linkages and bound calcium ions in dictating the pathway of the assembly process. We hope that this analysis may assist in determining optimal conditions for virus assembly, that may be exploited 
if viral capsids are assembled under laboratory conditions, for example as gene vectors in gene therapy [26].

\section{Acknowledgements}

RT has been supported by an EPSRC Advanced Research Fellowship and gratefully acknowledges funding for KME via the Leverhulme Trust Research Leadership Award F/00224/AE.

\section{References}

[1] N.A. Baker, D. Sept, S. Joseph, M.J. Holst, and J.A. McCammon, Electrostatics of nanosystems: Application to microtubules and the ribosome, Proc. Natl Acad. Sci. USA 98 (2001), pp. $10037-10041$.

[2] A. Bertolotti-Ciarlet, L.J. White, R. Chen, B.V. Prasad, and M.K. Estes, Structural requirements for the assembly of Norwalk virus-like particles, J. Virol. 76 (2002), pp. 4044-4055.

[3] B.R. Brooks, R.E. Bruccoleri, B.D. Olafson, D.J. States, S. Swaminathan, and M. Karplus, CHARMM a program for macromolecular energy, minimization, and dynamics calculations, J. Comput. Chem. 4 (1983), pp. 187-217.

[4] R.F. Bruinsma, W.M. Gelbart, D. Reguera, J. Rudnick, and R. Zandi, Viral self-assembly as a thermodynamic process, Phys. Rev. Lett. 90 (2003), p. 248101.

[5] D.L. Caspar and A. Klug, Physical principles in the construction of regular viruses, Cold Spring Harb. Sympos. Quant. Biol. 27 (1962), pp. 1-24.

[6] G. Christiansen, T. Landers, J. Griffith, and P. Berg, Characterization of components released by alkali disruption of simian virus 40, J. Virol. 21 (1977), pp. 1079-1084.

[7] K.M. ElSawy, L.S.D. Caves, and R. Twarock, Tubular structure of SV40 virus capsids, (2008), in preparation.

[8] K.M. ElSawy, M.K. Hodgson, and L.S.D. Caves, The physical determinants of the DNA conformational landscape: An analysis of the potential energy surface of single-strand dinucleotides in the conformational space of duplex DNA, Nucleic Acids Res. 33 (2005), pp. 5749-5762.

[9] D. Endres and A. Zlotnick, Model-based analysis of assembly kinetics for virus capsids or other spherical polymers, Biophys. J. 83 (2002), pp. 1217-1230.

[10] R.L. Garcea, Biologic constraint on modelling virus assembly, Comp. Math. Meth. Med. 9 (2008), pp. 257-264.

[11] B. Garcia-Moreno, J.J. Dwyer, A.G. Gittis, E.E. Lattman, D.S. Spencer, and W.E. Stites, Experimental measurement of the effective dielectric in the hydrophobic core of a protein, Biophys. Chem. 64 (1997), pp. 211-224.

[12] G. Gia and D.M.L. Maisuradze, Free energy landscape of a biomolecule in dihedral principal component space: Sampling convergence and correspondence between structures and minima, Proteins 67 (2007), pp. 569-578.

[13] H. Gohlke and D.A. Case, Converging free energy estimates: MM-PB(GB)SA studies on the proteinprotein complex Ras-Raf, J. Comp. Chem. 25 (2004), pp. 238-250.

[14] M.F. Hagan and D. Chandler, Dynamic pathways for viral capsid assembly, Biophys. J. 91 (2006), pp. $42-54$.

[15] M. Hemberg, S.N. Yaliraki, and M. Barahona, Stochastic kinetics of viral capsid assembly based on detailed protein structures, Biophys. J. 90 (2006), pp. 3029-3042.

[16] Li Hui, A.D. Robertson, and J.H. Jensen, Very fast empirical prediction and rationalization of protein pKa values, Proteins 61 (2005), pp. 704-721.

[17] K.I. Ishizu, H. Watanabe, S.I. Han, S.N. Kanesashi, M. Hoque, H. Yajima, K. Kataoka, and H. Handa, Roles of disulfide linkage and calcium ion-mediated interactions in assembly and disassembly of virus-like particles composed of simian virus 40 VP1 capsid protein, J. Virol. 75 (2001), pp. 61-72.

[18] C.C. Jao, M.K. Weidman, A.R. Perez, and E. Gharakhanian, Cys9, Cys104 and Cys207 of simian virus $40 \mathrm{Vpl}$ are essential for inter-pentamer disulfide-linkage and stabilization in cell-free lysates, J. Gen. Virol. 80 (1999), pp. 2481-2489.

[19] S.-n. Kanesashi, K.-i. Ishizu, M.-a. Kawano, S.-i. Han, S. Tomita, H. Watanabe, K. Kataoka, and H. Handa, Simian virus 40 VP1 capsid protein forms polymorphic assemblies in vitro, J. Gen. Virol. 84 (2003), pp. 1899-1905. 
[20] T. Keef, A. Taormina, and R. Twarock, Assembly models for Papovaviridae based on tiling theory, Phys. Biol. 2 (2005), pp. 175-188.

[21] T. Keef, A. Taormina, and R. Twarock, Classification of capped tubular viral particles in the family of Papovaviridae, J. Phys. Condens. Matter 18 (2006), p. S375.

[22] T. Keef, R. Twarock, and K.M. Elsawy, Blueprints for viral capsids in the family of Papovaviridae, J. Theor. Biol. (2008), to appear.

[23] A. Kosukegawa, F. Arisaka, M. Takayama, H. Yajima, A. Kaidow, and H. Handa, Purification and characterization of virus-like particles and pentamers produced by the expression of SV4O capsid proteins in insect cells, Biochim. Biophys. Acta 1290 (1996), pp. 37-45.

[24] R.C. Liddington, Y. Yan, J. Moulai, R. Sahli, T.L. Benjamin, and S.C. Harrison, Structure of simian virus 40 at 3.8A resolution, Nature 354 (1991), pp. 278-284.

[25] H.D. Nguyen, V.S. Reddy, and C.L. Brooks III, Deciphering the kinetic mechanism of spontaneous self-assembly of icosahedral capsids, Nano Lett. 7 (2007), pp. 338-344.

[26] A. Oppenheim, SV 40 assembly in vivo and in vitro, Comp. Math. Meth. Med. 9 (2008), pp. 265-276.

[27] E.B. Randolph and H. Michael, A new paradigm for parallel adaptive meshing algorithms, SIAM J. Sci. Comp. 22 (2000), pp. 1411-1443.

[28] D.C. Rapaport, Self-assembly of polyhedral shells: A molecular dynamics study, Phys. Rev. E 70 (2004), p. 051905.

[29] I. Rayment, T.S. Baker, D.L.D. Caspar, and W.T. Murakami, Polyoma virus capsid structure at $22.5 \AA$ resolution, Nature 295 (1982), pp. 110-115.

[30] D.M. Salunke, D.L. Caspar, and R.L. Garcea, Self-assembly of purified polyomavirus capsid protein VP1, Cell 46 (1986), pp. 895-904.

[31] D.M. Salunke, D.L. Caspar, and R.L. Garcea, Polymorphism in the assembly of polyomavirus capsid protein VP1, Biophys. J. 56 (1989), pp. 887-900.

[32] D. Sept, N.A. Baker, and J.A. McCammon, The physical basis of microtubule structure and stability, Protein Sci. 12 (2003), pp. 2257-2261.

[33] D. Sitkoff, K.A. Sharp, and B. Honig, Accurate calculation of hydration free energies using macroscopic solvent models, J. Phys. Chem. 98 (1994), pp. 1978-1988.

[34] T. Stehle, S.J. Gamblin, Y. Yan, and S.C. Harrison, The structure of simian virus 40 refined at $3.1 \AA$ resolution, Structure 4 (1996), pp. 165-182.

[35] J.M. Swanson, R.H. Henchman, and J.A. McCammon, Revisiting free energy calculations: $A$ theoretical connection to MM/PBSA and direct calculation of the association free energy, Biophys. J. 86 (2004), pp. 67-74.

[36] E.C. Thomas, Disulphide bonds and protein stability, Bioessays 8 (1988), pp. 57-63.

[37] R. Twarock, Mathematical models for tubular structures in the family of Papovaviridae, Bull. Math. Biol. 67 (2005), pp. 973-987.

[38] R. Twarock, Mathematical virology: A novel approach to the structure and assembly of viruses, Philos. Trans. R. Soc. A 364 (2006), pp. 3357-3373.

[39] R. Zandi, D. Reguera, R.F. Bruinsma, W.M. Gelbart, and J. Rudnick, Origin of icosahedral symmetry in viruses, Proc. Natl Acad. Sci. U S A 101 (2004), pp. 15556-15560.

[40] A. Zlotnick, R. Aldrich, J.M. Johnson, P. Ceres, and M.J. Young, Mechanism of capsid assembly for an icosahedral plant virus, Virology 277 (2000), pp. 450-456. 


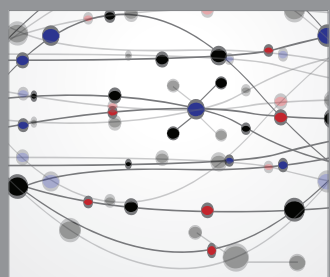

The Scientific World Journal
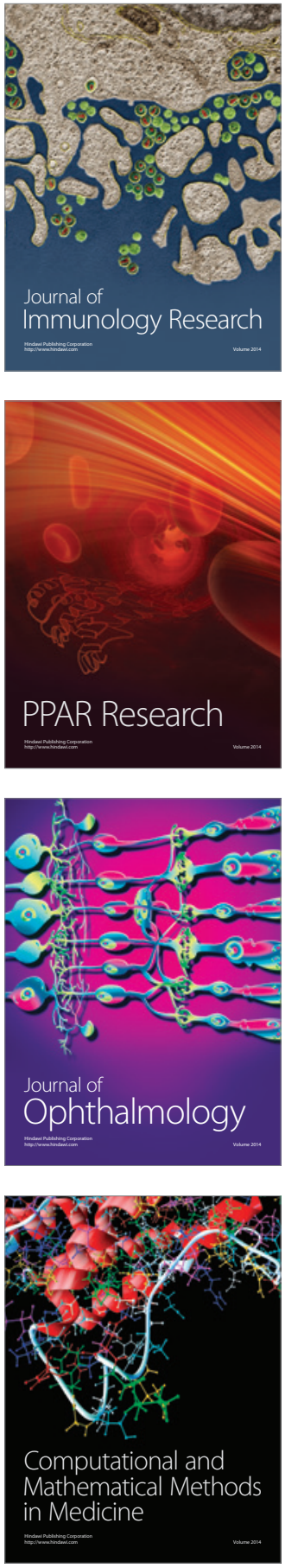

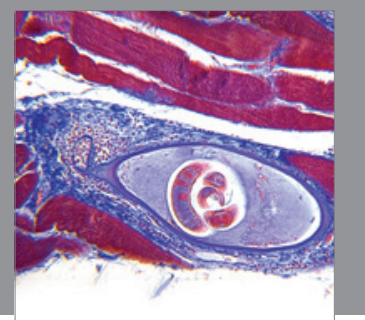

Gastroenterology

Research and Practice
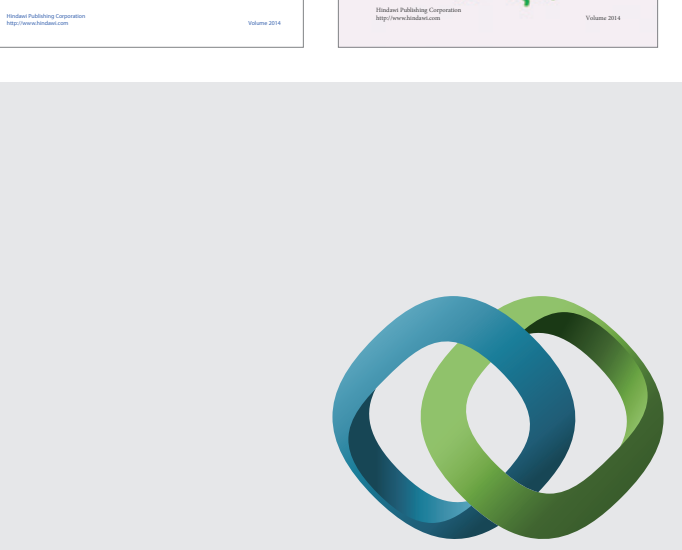

\section{Hindawi}

Submit your manuscripts at

http://www.hindawi.com
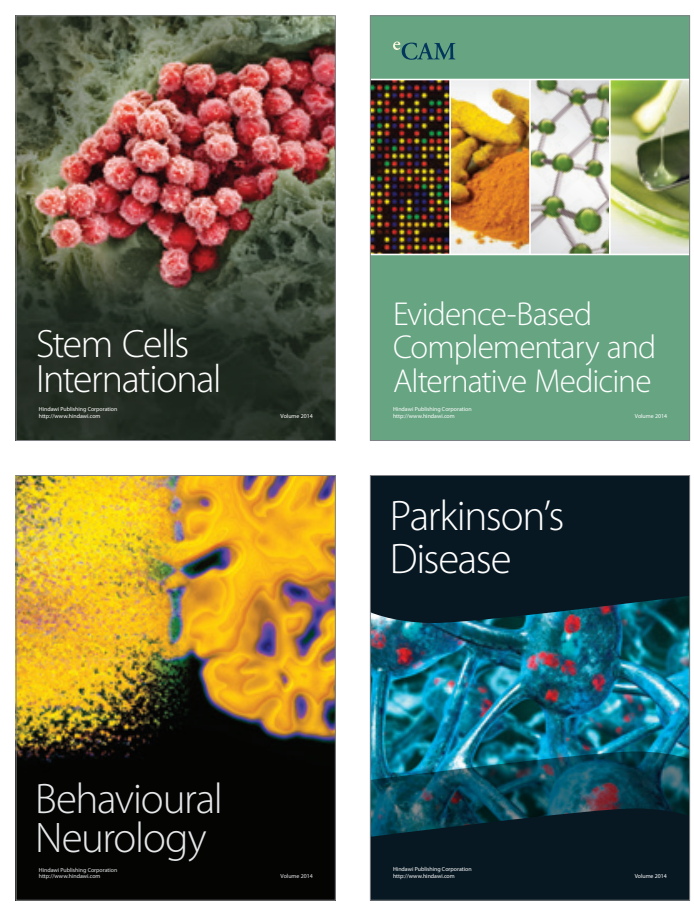

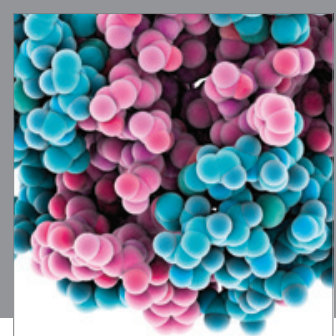

Journal of
Diabetes Research

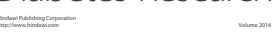

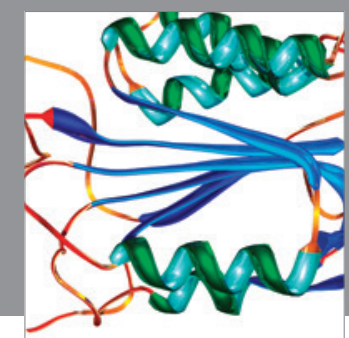

Disease Markers
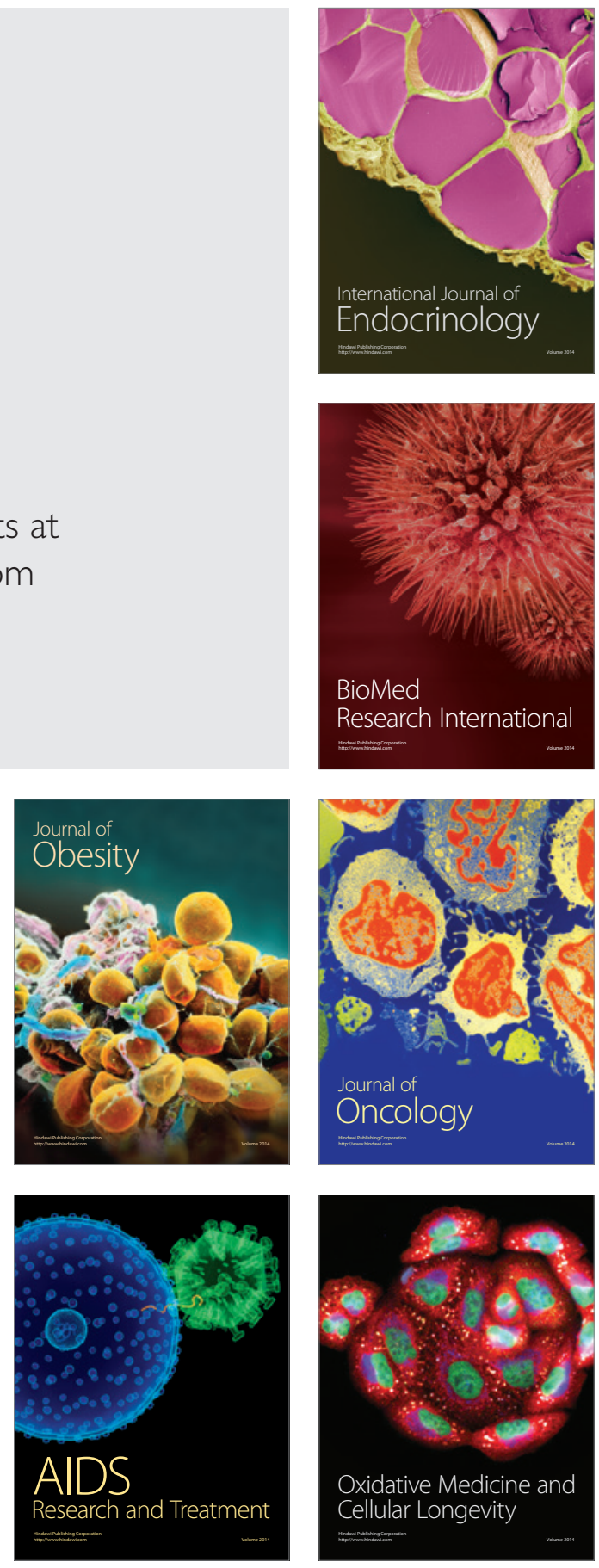